\title{
Hi-def memories of lo-def scenes
}

\author{
Jose Rivera-Aparicio ${ }^{1} \cdot$ Qian $_{\mathrm{Yu}^{1}} \cdot$ Chaz Firestone $^{1}$ (D)
}

Accepted: 6 October 2020 / Published online: 14 January 2021

(C) The Psychonomic Society, Inc. 2021

\begin{abstract}
The study of visual memory is typically concerned with an image's content: How well, and with what precision, we can recall which objects, people, or features we have seen in the past. But images also vary in their quality: The same object or scene may appear in an image that is sharp and highly resolved, or it may appear in an image that is blurry and faded. How do we remember those properties? Here six experiments demonstrate a new phenomenon of "vividness extension": a tendency to (mis)remember images as though they are "enhanced" versions of themselves - that is, sharper and higher quality than they actually appeared at the time of encoding. Subjects briefly saw images of scenes that varied in how blurry they were, and then adjusted a new image to be as blurry as the original. Unlike an old photograph that fades and blurs, subjects misremembered scenes as more vivid (i.e., less blurry) than those scenes had actually appeared moments earlier. Follow-up experiments extended this phenomenon to saturation and pixelation - with subjects recalling scenes as more colorful and resolved - and ruled out various forms of response bias. We suggest that memory misrepresents the quality of what we have seen, such that the world is remembered as more vivid than it is.
\end{abstract}

Keywords Scene perception $\cdot$ Memory $\cdot$ Vividness $\cdot$ Boundary extension

\section{Introduction}

What's in a picture? The images we see have content, such as objects, places, events, and people; a photograph, for example, might show two friends strolling on a beach. However, images vary not only in their content, but also in their quality: A photograph of the very same beach-walk may be sharp, resolved, and saturated; or it could be blurry, grainy, or faded - and this too is a property of images that we may see and encode. Research on visual memory is typically concerned with memory for the former kinds of details - the capacity and precision of our ability to recall contentful information such as which people, objects, events, places, colors, or shapes were present in an image. But what about the latter kinds of details? How do we remember the quality of the images we see?

Suppose, for example, that you see a blurry photograph, grainy television broadcast, or faded piece of artwork, and you

Chaz Firestone

chaz@jhu.edu

1 Department of Psychological and Brain Sciences, Johns Hopkins University, 3400 N Charles St, Baltimore, MD 21218, USA attempt to recall not what the images were about, but instead how blurry, grainy, or faded they were, regardless of their content. Is memory for such properties accurate? Or might such memories show biases, misrepresenting the quality of the images we see? Here, we explore this question by investigating memory for image quality.

\section{Are memories better or worse than the real thing?}

What kind of bias might there be for memories of image quality? On one hand, memories decay (in that stored information becomes harder to retrieve over time), and this might produce a corresponding "decay" in the quality attributed to those features. In that case, as memories of a photograph or broadcast fade into the past, we might remember the images themselves as having been grainier and more faded. Indeed, it has recently been suggested that memories "literally fade," such that images may be estimated as less bright or salient when they are recalled from memory than when they are viewed online (Cooper, Kensinger, \& Ritchey, 2019). This prediction not only seems subjectively intuitive, but it could also naturally emerge from sensory recruitment models of working memory, on which such memories are essentially reactivations of sensory states (Harrison \& Tong, 2009; 
Serences et al., 2009). Since such reactivations are subject to decay, we might expect to misremember images as lower quality than they really were, if we re-enter a lower-quality state at recall. (Or, we might simply remember the quality of such experiences accurately, if the revisited sensory state were sufficiently well preserved.)

On the other hand, memories often fill in details that were not actually present during encoding. One salient example of this process is "boundary extension," wherein scenes are misremembered as being wider angled and having additional objects and details that are typical of those scenes (Intraub \& Richardson, 1989; Park et al., 2007). Such phenomena might imply a bias toward remembering images as higher quality. This prediction - that images could be remembered as though they were "enhanced" versions of themselves - may seem less intuitive; after all, memory is often characterized as a weaker or less vivid form of perception. At the same time, a tendency towards "vividness" might be consistent with models of memory that incorporate prior knowledge or schemas in reconstructing what was seen (Bartlett, 1932; see also Brewer \& Treyens, 1981; Huttenlocher, Hedges, \& Duncan, 1991), including more recent incarnations of such models that characterize the role of prior knowledge in terms of Bayesian updating (Bae et al., 2015; Hemmer \& Steyvers, 2009; Persaud \& Hemmer, 2016). Indeed, there may even be anecdotal evidence for such a tendency: For example, when one returns to a famous television broadcast or childhood video game, one often observes that the broadcast or game seems lower quality than one remembered (was the footage really that grainy?), as if the images are remembered in "higher definition" than they really appeared (perhaps because the mind added extra image details based on prior knowledge).

Do either of these directional biases occur? Here, we ask whether memory - specifically, working or short-term memory - systematically misrepresents the quality of what we have seen. Subjects saw degraded scenes (e.g., blurry, pixelated, or desaturated images), and were then asked to reproduce such degradation in a new image. To foreshadow the key result, we observed a tendency towards vividness, wherein subjects remembered scenes as being (on average) less blurry, more resolved, and even more colorful than they had actually appeared moments before - a phenomenon we call "vividness extension."

\section{Experiment 1: Vividness extension for blur}

As an initial test of vividness extension, we asked subjects to remember and reproduce the amount of blur in an image that was seen moments earlier. Are such memories distorted with respect to vividness?

\section{Method}

\section{Participants}

One hundred subjects were recruited online through Amazon Mechanical Turk. (For discussion of this subject pool's reliability, see Crump, McDonnell, \& Gureckis, 2013.) Here, this sample size was chosen simply because it seemed large; later experiments used and pre-registered this same sample size (except Experiment 6, which used a larger pre-registered sample ); we also report power analyses given those sample sizes.

\section{Stimuli and procedure}

The experiment consisted of 50 trials, each proceeding in the same way (Fig. 1). On each trial, subjects saw one of 50 natural scenes $(256 \times 256$ pixels $(\mathrm{px}))$ drawn from a range of categories (e.g., beach, mountain, street; Oliva \& Torralba, 2001). Each presented scene was distorted by a different amount of Gaussian blur: On each trial, one scene appeared at a random blur value (0- to 20-px radius), and remained on the display for $2 \mathrm{~s}$. Next, it disappeared for $2 \mathrm{~s}$ (during which the screen was blank), until the same scene reappeared in the same location, but this time at a different random blur value (also drawn from the 0- to 20-px range), with a slider located above it. Subjects were instructed to "adjust this image to be exactly as blurry as the one you just saw," and used the slider to alter the blur level of this second scene until they believed it matched the blur of the earlier scene. (The "sign" of the slider was randomly assigned to each subject, such that for approximately half the subjects, the slider's left end was the blurry end and the right end was the clear end, and for the other half of subjects, right was the blurry end and left was clear.) After estimation, the adjustable scene disappeared, and the next trial began after $2 \mathrm{~s}$.

Subjects were excluded if their blur estimates were not significantly correlated with the true blurriness of the images over the span of 50 trials (which may have indicated a lack of understanding or engagement), or if they failed to contribute a complete dataset. (These same exclusion criteria were used for every experiment here, and were pre-registered for Experiments 3-6.)

Readers can experience the task for themselves at: https:// perceptionresearch.org/vividness. Additionally, all data, analyses, materials (i.e., stimuli and experiment code), and pre-registrations (for Experiments 3-6) are available at https://osf.io/wjtnx.

\section{Results and discussion}

Subjects tended to overestimate the clarity of the remembered images. In other words, subjects adjusted the test scenes to be less blurry than the true blurriness of those scenes, estimating 


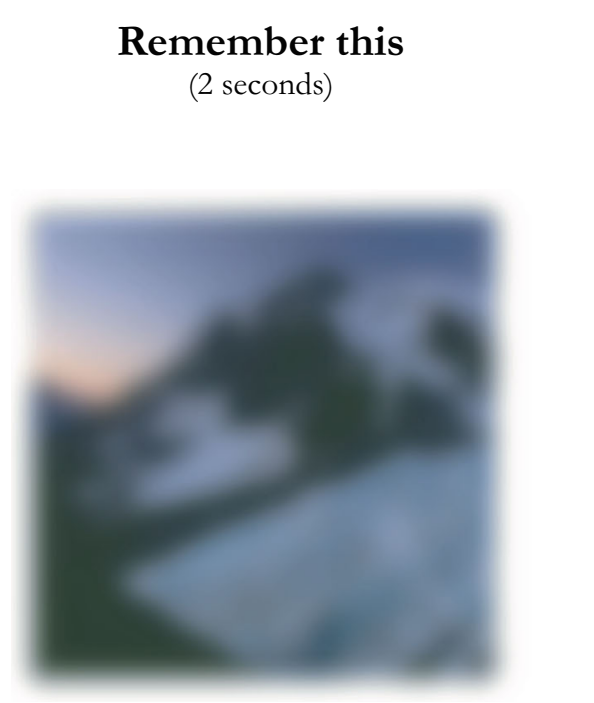

I
I
I
I
I
I
I
I
I
I
I
I
I
I
I

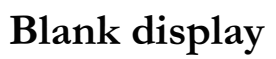

(2 seconds)

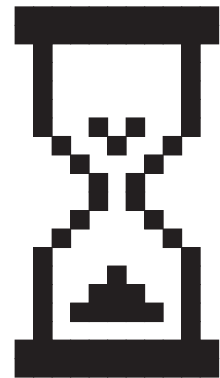

How blurry was it?

(adjust until satisfied)

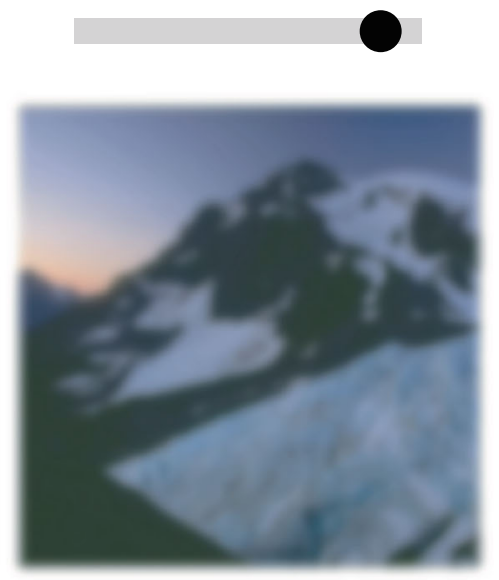

\section{Time}

Fig. 1 On each trial of Experiment 1, subjects were shown a scene that was distorted by a random amount of blur. After a delay (during which the screen was blank), the same scene reappeared in the same location, but this time at a different random blur value. Subjects were instructed to use a slider to "adjust this image to be exactly as blurry as the one you just

the blur-radius at an average of $8.54 \mathrm{px}$, which significantly differed from the true average blur-radius of $10 \mathrm{px}, t(80)=$ $10.62, \mathrm{SE}=0.14 \mathrm{px}, d=1.18 ; 95 \% \mathrm{CI}_{\text {bias }}=1.46[1.19,1.73]$, $p<.001$; Fig. 2a. (Four subjects were excluded for failing to contribute a complete dataset; of the 96 subjects remaining, a further 15 were excluded for poor data quality, using the predetermined exclusion criteria. However, no effects reported here depended in any way on these exclusions; in other words, all effects remained statistically significant regardless of whether these data were included - and this is true for every experiment reported in this paper.)

Figure 2a also breaks down the effect by individual blur values. As can be seen, even though vividness extension was observed overall, this trend was not evident at every single presented blur value; indeed, for the clearest (i.e., least blurry) images, there was actually a blur bias in recall, not a vividness bias. However, this pattern can be explained simply by the estimation constraints imposed on subjects, which allowed subjects to respond only within the same 0- to 20-px blur range that the images appeared in. For example, consider the most extreme case: an image shown in perfect clarity (i.e., 0 px blur). For that image, it was only possible to observe a blurbias, unless every single subject always estimated its blur with perfect accuracy; instead, the fact that this sort of trial (and others toward the "clear" end of the spectrum) show a blurbias can be explained simply by a tendency to respond saw," with no time pressure. A similar design was used in the other experiments, though see text for details about how they differed. Readers can experience this task for themselves at https:// perceptionresearch.org/vividness

towards the center of the estimation scale (either because subjects avoid extreme responses, or because some subjects sometimes responded randomly or uncarefully), in a way that causes a parallel regression-to-the-mean effect (i.e., to the mean of possible slider values). By contrast, the overall tendency towards vividness, collapsing across all blur values, seems to go beyond the particular constraints of the scale. Indeed, this can also be seen by noting that the estimate for the "halfway" point of the scale (10 px) was a blurriness value of $8.9 \mathrm{px}$ (i.e., less blurry, or more vivid), suggesting that the tendency we observed occurred over and above any mean regression. (See Experiment 6 for another analysis that further explores this pattern.)

These results thus lend initial support to the hypothesis that subjects remember scenes as being more vivid than they really were - a phenomenon of vividness extension.

\section{Experiments 2-4: Vivid memories or biased responses?}

Do we truly remember images as being more vivid? Perhaps, instead, subjects prefer vivid scenes and simply adjusted the test scenes to match this preference (e.g., because they enjoy looking at vivid images, or would like the images to be vivid, etc.; Firestone \& Scholl, 2015). Or perhaps subjects reasoned 


\section{Experiment 1 (Blur)}

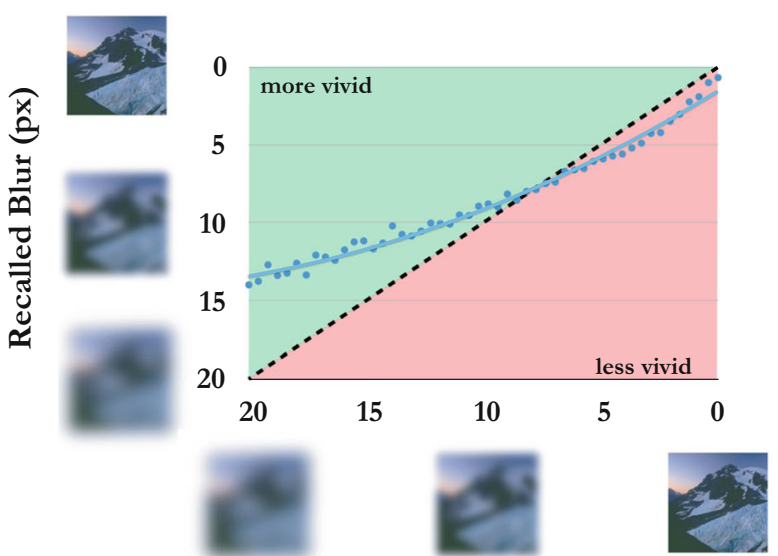

Actual Blur (px)

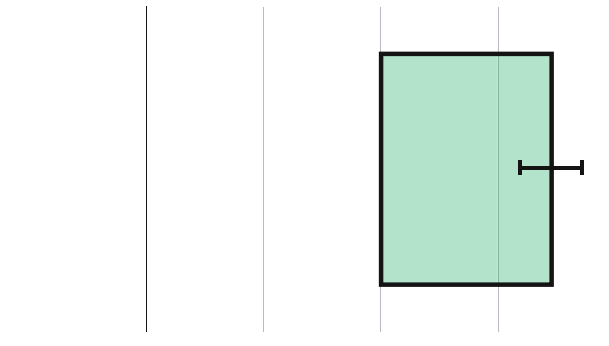

$\longleftarrow$ blurrier

clearer

\section{Vividness Bias (px)}

b

Experiment 5 (Pixelation)

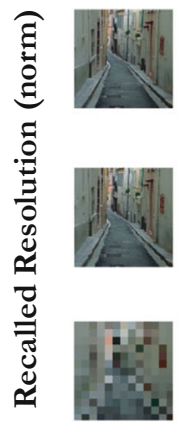

$$
1
$$

0.75

more vivid
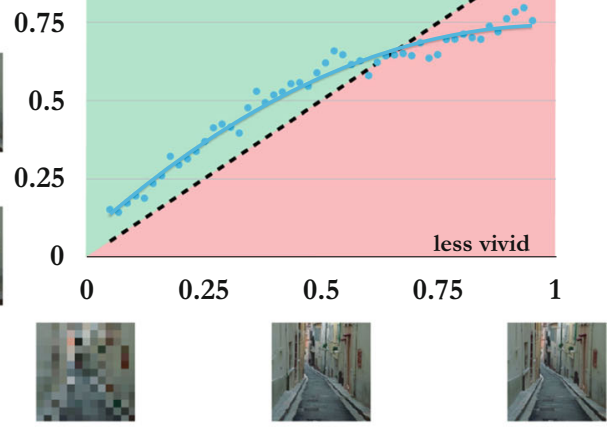

Actual Resolution (norm)

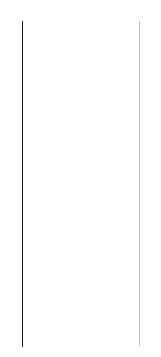

more pixelated

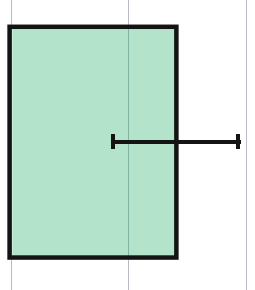

Vividness Bias (norm)

C

\section{Experiment 6 (Saturation)}

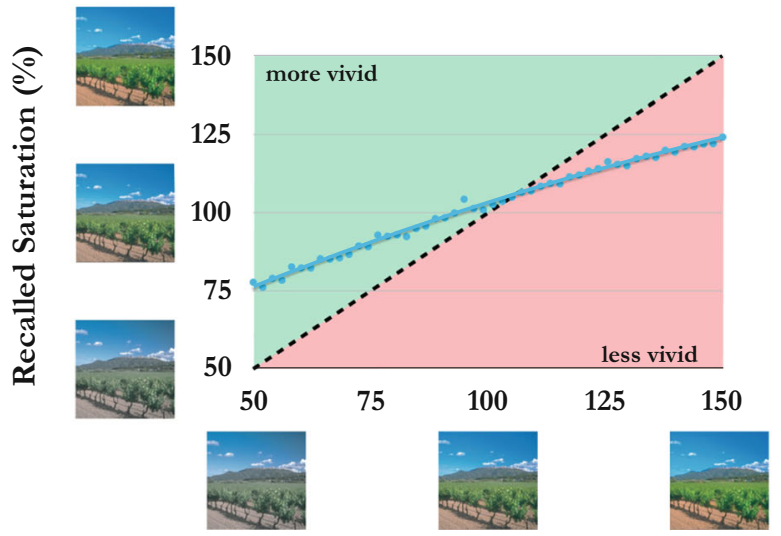

Actual Saturation (\%)

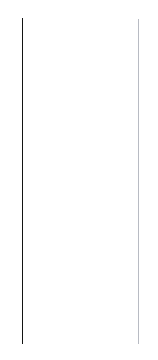

less saturated

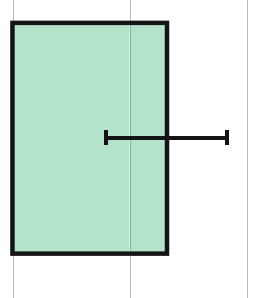

more saturated

Vividness Bias (\%) 
4 Fig. 2 Subjects recalled recently seen images as more vivid than they really were. This pattern held across (a) blur, (b) pixelation, and (c) saturation. Error bars represent \pm 1 standard error of the mean difference between estimated vividness (as recalled) and actual vividness (as presented)

their way into believing the scene was clearer - a kind of "hindsight bias" (Roese \& Vohs, 2012) produced by learning the scene's true, unblurred identity upon exploring with the slider at test (which may even alter appearance; Perez, Cook, $\&$ Peterson, 2020). Experiments 2-4 ruled out these possibilities.

\section{Method}

Experiments 2-4 proceeded in the same way as Experiment 1, except as noted here. 300 subjects participated (100 in each experiment). For Experiments 3 and 4, the hypothesis, sample size, and analysis plan (including exclusion criteria) were preregistered. Assuming the same effect size and exclusion rate as in Experiment 1, each of these experiments had greater than 99\% power to detect vividness extension.

In Experiment 2, the only difference from Experiment 1 was that the target scene was left on the screen at test, such that the blurriness estimation was made during online viewing instead of from memory. In other words, subjects were given the same instructions as in Experiment 1, except they were asked to adjust the blurriness of an image to match not some previously remembered image, but instead a copy of that image that was still easily visible on the screen. The purpose of this modification was to rule out a more general response bias favoring vividness; if the results of Experiment 1 arose simply because subjects enjoy setting the slider to the vivid end, then that bias should apply in this case too (à la the El Greco fallacy; Firestone, 2013; Firestone \& Scholl, 2014; Valenti \& Firestone, 2019). However, if the vividness extension effect from Experiment 1 is explained by a genuine memory distortion, then any effects here in Experiment 2 should be smaller or non-existent.

In Experiment 3, the only difference from Experiment 1 was that the to-be-recalled blurry scene was different than the adjustable scene used for estimation (Fig. 3a). In other words, subjects were given the same instructions from Experiment 1, i.e., to reproduce the amount of blur they had just seen, but using a different token scene image. The purpose of this modification was to test whether the vividness extension effect was merely due to acquiring new information about the true identity of the adjustable scene while moving the slider. For example, in Experiment 1, after seeing a blurry "beach" scene at encoding and then moving the slider around at test, subjects had the opportunity to learn all sorts of finer details about the scene, which may have caused a kind of "hindsight bias" (Roese \& Vohs, 2012), such that subjects believed they knew these finer details all along. By making the blurry target scene and adjustable scene different (e.g., a blurry beach at encoding, and an adjustably blurry forest at test), we prevented participants from gaining this new information about the beach, such that manipulating the slider shouldn't alter their representation of the previously seen image. In that case, observing vividness extension even in Experiment 3 would suggest that hindsight bias could not fully explain the results.

Finally, Experiment 4 combined the designs of Experiments 2 and 3 into a single within-subjects experiment. Subjects saw two kinds of trials, randomly interleaved: "perception" trials (as in Experiment 2), and "memory" trials (as in Experiment 3), both involving an adjustable test image that had a different identity than the target image. In other words, in a "perception" trial, subjects might adjust a forest scene to match the blur of a beach that was present on the display during test; and in a "memory" trial, subjects might adjust a forest scene to match the blur of a beach that had been presented moments earlier. This allowed us to compare tendencies toward vividness in perception versus in memory, in the same individual subjects, tested under the same circumstances.

These experiments thus ask whether vividness extension is a memory distortion per se, or whether it can be explained by other factors.

\section{Results and discussion}

All three experiments supported a genuine memory distortion, rather than a response bias or hindsight bias.

In Experiment 2, where the blurry image remained onscreen at test, a very small vividness extension effect emerged (9.67 px vs. $10 \mathrm{px}, \mathrm{t}(95)=5.31, \mathrm{SE}=0.06 \mathrm{px}, d=0.54,95 \% \mathrm{CI}_{\text {bias }}=0.33$ $[0.21,0.45], \mathrm{p}<.001)$; however, this effect was considerably smaller than in Experiment 1 - and significantly so, $8.54 \mathrm{px}$ vs. $9.67 \mathrm{px}, t(175)=7.87,95 \% \mathrm{CI}_{\text {difference }}=1.13[0.83,1.42]$, $p<.001$. This suggests that, even if subjects do prefer to look at clearer scenes, this can't explain all (or even most) of the original vividness extension results.

In Experiment 3, vividness extension emerged again (Fig. $3 b)$. Subjects overestimated the clarity of remembered images, even when the estimation procedure offered no new information about details in the remembered scene, and even under the unusual circumstance of matching a property of one scene to that same property in a completely different scene. The average blur estimate was $9.40 \mathrm{px}$, which significantly differed from the true average blur of $10 \mathrm{px}, t(73)=3.98, \mathrm{SE}=$ $\left.0.15 \mathrm{px}, d=.46,95 \% \mathrm{CI}_{\text {bias }}=0.60[0.30,0.90], p<.001\right)$. Note that this result is in some sense opposite the one that would be expected if scene knowledge altered perception of blur in this experiment (e.g., as reported by Perez, Cook, \& Peterson, 2020, who suggest that familiar objects look less blurry than unfamiliar ones). In the present experiment, the ability to explore the test scene and determine its identity (by adjusting its 


\section{Experiment 3 (Different Images)}

a

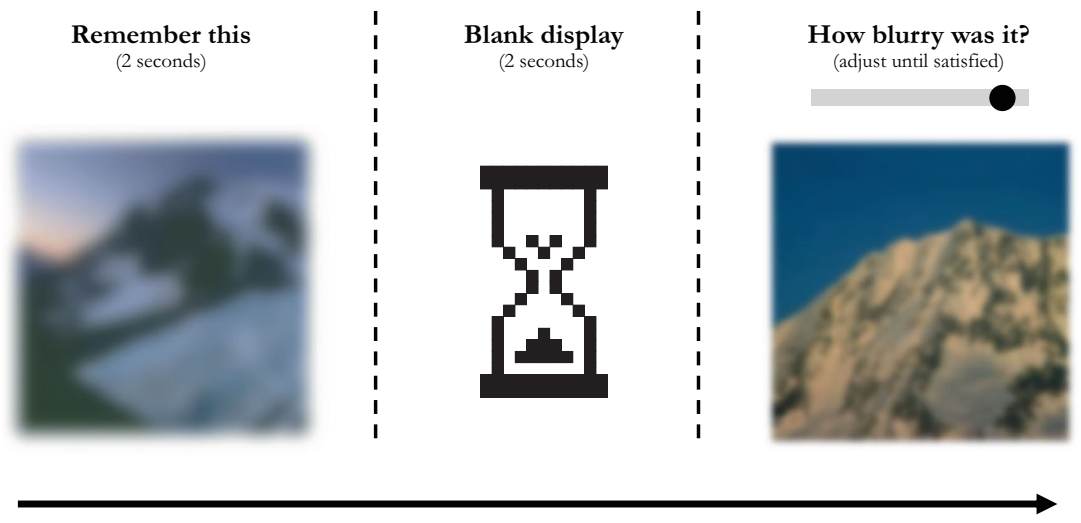

Time

b

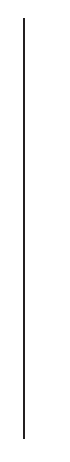

$-2$

blurrier

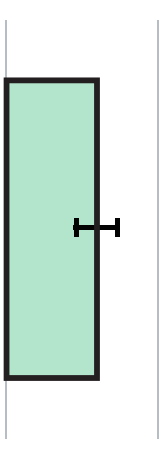

0

1

2

clearer

\section{Vividness Bias (px)}

Fig. 3 a In Experiment 3, subjects recalled the estimated the blurriness of one image by adjusting the blurriness of a different image. $\mathbf{b}$ Even under these conditions, a tendency towards vividness emerged. Error bars

blur) should have made that scene (i.e., the test scene) appear less blurry, which in turn should have made subjects select a blurrier match to compensate, not a clearer one. So, this result suggests that the overall tendency towards vividness cannot be explained by an impact of high-level scene knowledge on perceived blur.

Finally, Experiment 4 allowed for a direct comparison of perception and memory in the same subjects. And indeed, vividness extension for memory (average blur estimate: 9.00 px) was stronger than for perception (average blur estimate: $9.28 \mathrm{px}), t(91)=2.47, \mathrm{SE}=0.11 \mathrm{px}, d=.26,95 \% \mathrm{CI}_{\text {difference }}=$ $0.28[0.05,0.51], p=.015$. This result thus served as a replication of each of the patterns observed in Experiments 2 and 3: represent \pm 1 standard error of the mean difference between estimated vividness (as recalled) and actual vividness (as presented)

Vividness extension is observed even when the test image differs from the target image, and it is stronger when image quality is recalled from memory rather than judged online.

These results suggest that the phenomenon of vividness extension goes beyond response bias or hindsight bias, and in fact reflects a genuine distortion of memory for vividness.

\section{Experiments 5-6: Vividness beyond blur - resolution and saturation}

Does vividness extension generalize beyond a scene's clarity? Experiments 5-6 explored this question for resolution and 
saturation, asking whether subjects misremember images as less pixelated and more colorful.

\section{Method}

Experiments 5 and 6 proceeded in the same way as Experiment 1, except as noted here. 100 subjects participated in Experiment 5, and 500 subjects participated in Experiment $6 .^{1}$ Both experiments were pre-registered.

\section{Stimuli and procedure}

In Experiment 5, the type of degradation applied to the images was pixelation. The scenes shown at the time of encoding were degraded to various levels of pixelation (which we report here in terms of normalized degree of pixelation, between 0 and 1). Then, at test, participants were asked to match the amount of pixelation (rather than the amount of blur as in previous experiments). One important property of the pixelation space as a test of vividness extension is that, whereas the blurry images from Experiment 1 ended up having a larger spatial envelope (since the blur bled out beyond the boundary of the original image), this was not true for highly pixelated images, which maintained their precise dimensions even as they became more pixelated. Moreover, the "pixelation space" as implemented here had different nonuniformities than the previous "blur space." For example, with blurry images, just a tiny bit of blur applied to an otherwise crisp image was quite subjectively noticeable, whereas a similar move of the slider at the blurrier end of the spectrum was perhaps not so noticeable; for pixelation, this was reversed, such that a small amount of pixelation applied to a crisp image was not so subjectively noticeable, whereas equal steps at the highly pixelated end of the spectrum were very noticeable. As a result, pixelation differed from blur not only in being a new property, but also a new kind of degradation space in which to explore vividness extension.

In Experiment 6, the only difference relative to Experiment 1 was that the distortion applied to the images involved saturation, such that the scenes shown at the time of encoding could vary from $50 \%$ to $150 \%$ of their native saturation. At the time of making their estimate, participants matched the amount of saturation in the scene instead of the amount of blur (Experiment 1) or pixelation (Experiment 5), using the same $50-150 \%$ space.

\footnotetext{
${ }^{1}$ Assuming the same effect size and exclusion rate as Experiment 1, Experiment 5 had over $99 \%$ power to detect a vividness extension effect of similar size. By contrast, a pilot version of Experiment 6, which obtained a marginally significant effect, suggested that 500 subjects would achieve $98 \%$ power to detect vividness extension for saturation; we thus pre-registered this sample size for Experiment 6. As is clear from the results, however, the effect in Experiment 6 ended up being comparable to other experiments, such that 500 subjects turned out to be far more than was required to detect the relevant effect.
}

\section{Results and discussion}

Vividness extension was observed for both pixelation and saturation; in other words, subjects selected more resolved (i.e., less pixelated) images in Experiment 5, and more colorful (i.e., less desaturated) images in Experiment 6, compared to the true vividness of those scenes viewed moments earlier.

For Experiment 5, estimated pixelation was .53, which differed from the true average pixelation of $.5, t(84)=3.52$, $\mathrm{SE}=0.008, d=0.38,95 \% \mathrm{CI}_{\text {bias }}=0.03[0.015,0.045]$, $p<.001$; Fig. 2 b.

For Experiment 6, estimated saturation was $101.96 \%$, which significantly differed from the true average saturation of $100 \%$, $t(378)=5.74, \mathrm{SE}=0.31 \%, d=0.29,95 \% \mathrm{CI}_{\text {bias }}=1.96[1.36$, 2.56], $p<.001$; Fig. 2c. This result goes even further beyond the previous experiments in that scenes were essentially recalled as "supersaturated" - not only more saturated than they had actually appeared on the display (as in our earlier experiments), but also more saturated than such images would natively appear (since the average saturation level estimated by subjects was above $100 \%$ ). This result may thus further suggest that vividness extension is not merely a bias towards "realism," since in this case that might have predicted no effect at all.

Finally, since Experiment 6 included values both below and above the "true" saturation value, this experiment also allows us to conduct a regression to probe the nature of this tendency. A linear regression on the data from Experiment 6 revealed a positive intercept $(54.19 \%$ saturation, $\mathrm{SE}=0.53$, $95 \% \mathrm{CI}=[53.15,55.23], p<.001)$ and a slope below $1(0.48$, $\left.\mathrm{SE}=0.005,95 \% \mathrm{CI}=[0.47,0.49], p<.001, \mathrm{R}^{2}=0.32\right)$. These results confirm what is also visually apparent from looking at Fig. 2: That there is an overall tendency towards vividness, but that not all values are shifted towards vividness; in other words, there is also regression to the mean (in this case, the mean vividness and slider values), perhaps reflecting constraints on the response space (e.g., at the extremely "vivid" ends, there is not much room to move upwards). Still, the positive intercept suggests that even the few saturation values that are shifted away from vividness are weaker and less numerous than those shifted towards vividness, such that there is still an overall tendency towards vividness when considering the entire space of images and responses.

These results suggest that the phenomenon of vividness extension is robust and general, and that it affects various kinds of image quality - not only blur, but also resolution and saturation.

\section{General discussion}

A pervasive and intuitive assumption about memory is that it is merely a faded or degraded version of the vivid perceptual experiences it encodes - "by its very nature weaker 
and fainter than a [sensory] impression" (Hume, 1739/ 1978, p.72-73). By contrast, subjects here misremembered their environment as more vivid than it had been in reality. This "vividness extension" effect could not be explained by biased responding, went beyond regression to the mean, and generalized across several forms of vividness - including blur, saturation, and resolution.

\section{More than a game}

Our results were inspired by the anecdotal surprise that is sometimes observed with respect to the vividness of various media (e.g., television broadcasts and video games); however, these results go beyond their anecdotal inspiration to bear on broader questions about the relationship between memory and perception.

First, these results are perhaps counterintuitive from the perspective that visual short-term memories are essentially "reactivations" of sensory states. Since the traces of such sensory states decay over time, one might have expected that memories would represent past experiences as more faded or grainy (or at least faithfully preserved) - not more vivid (Cooper et al., 2019). Though we do not think these results reject or refute sensory recruitment models of working memory (see Xu, 2017, for a more in-depth discussion), they do suggest that working memory goes beyond simply "reexperiencing" images from the past.

Second, these results may have implications for other debates that rely on (and perhaps assume accuracy about) memory for the vividness of our experiences. For example, many currently debated issues in perception (and even in the philosophy thereof; Siegel \& Byrne, 2017) concern the "richness" of perceptual experience - for example, whether dreams or hallucinations are as rich and vivid as day-to-day perception (Ichikawa, 2009), and more generally how reliable memory for vividness is (Engelhard et al., 2019). Such arguments, when relying only on introspection, could be subject to vividnessextension-like distortions: We might believe our dreams and hallucinations are as rich as our waking lives only because we misremember those experiences as more vivid than they really were. Participants in such debates, then, could make sure to consider the possibility that their memory for vividness is distorted and/or unreliable.

Finally, these results dovetail nicely with accounts that treat memory not as a reinstantiation of perception, but rather as a structured description of what we have seen - one that aims to represent the content or reference of an image, rather than storing a faithful copy of the image for later revisiting (Brady, Konkle, \& Alvarez, 2011; Gallistel \& King, 2009; Park et al., 2011). Indeed, just as boundary extension adds details outside remembered scenes (Intraub \& Richardson, 1989; Park et al., 2007; cf.
Bainbridge \& Baker, 2020), vividness extension suggests that a similar process operates even within a scene itself. In other words, our minds store our experiences as though they were in "hi-def" at every location within the images we see.

Open Practices Statement All data and materials supporting these experiments are available at https://osf.io/wjtnx. Experiments 3-6 were pre-registered.

\section{References}

Bae, G. Y., Olkkonen, M., Allred, S. R., \& Flombaum, J. I. (2015). Why some colors appear more memorable than others: A model combining categories and particulars in color working memory. Journal of Experimental Psychology: General, 144, 744.

Bainbridge, W. A., \& Baker, C. I. (2020). Boundaries extend and contract in scene memory depending on image properties. Current Biology, 30, 537-543.

Bartlett, F. C. (1932). Remembering: A study in experimental and social psychology. Cambridge: Cambridge University Press.

Brady, T. F., Konkle, T., \& Alvarez, G. A. (2011). A review of visual memory capacity: Beyond individual items and toward structured representations. Journal of Vision, 11, 4:1-34.

Brewer, W. F., \& Treyens, J. C. (1981). Role of schemata in memory for places. Cognitive Psychology, 13, 207-230.

Cooper, R. A., Kensinger, E. A., \& Ritchey, M. (2019). Memories fade: The relationship between memory vividness and remembered visual salience. Psychological Science. Published online ahead of print.

Crump, M. J., McDonnell, J. V., \& Gureckis, T. M. (2013). Evaluating Amazon's Mechanical Turk as a tool for experimental behavioral research. PLoS One, 8, e57410.

Engelhard, I. M., McNally, R. J., \& van Schie, K. (2019). Retrieving and modifying traumatic memories: Recent research relevant to three controversies. Current Directions in Psychological Science, 28, 91-96.

Firestone, C. (2013). On the origin and status of the "El Greco fallacy". Perception, 42, 672-674.

Firestone, C., \& Scholl, B. J. (2014). "Top-down" effects where none should be found: The El Greco fallacy in perception research. Psychological Science, 25, 38-46.

Firestone, C., \& Scholl, B. J. (2015). When do ratings implicate perception vs. judgment? The "overgeneralization test" for top-down effects. Visual Cognition, 23, 1217-1226.

Gallistel, C. R., \& King, A. P. (2009). Memory and the computational brain: Why cognitive science will transform neuroscience. John Wiley \& Sons. https://doi.org/10.1002/9781444310498.

Harrison, S. A., \& Tong, F. (2009). Decoding reveals the contents of visual working memory in early visual areas. Nature, 458, 632-635.

Hemmer, P., \& Steyvers, M. (2009). A Bayesian account of reconstructive memory. Topics in Cognitive Science, 1, 189-202.

Hume, D. (1739/1978). A Treatise of Human Nature (Book 1). Oxford: Oxford University Press.

Huttenlocher, J., Hedges, L. V., Duncan, S. (1991). Categories and particulars: Prototype effects in estimating spatial location. Psychological Review, 98, 352-376.

Ichikawa, J. (2009). Dreaming and imagination. Mind and Language, 24, $103-121$ 
Intraub, H., \& Richardson, M. (1989). Wide-angle memories of close-up scenes. Journal of Experimental Psychology: Learning, Memory, and Cognition, 15, 179-187.

Oliva, A., \& Torralba, A. (2001). Modeling the shape of the scene: A holistic representation of the spatial envelope. International Journal of Computer Vision, 42, 145-175.

Roese, N. J., \& Vohs, K. D. (2012). Hindsight bias. Perspectives on Psychological Science, 7, 411-426.

Park, S., Brady, T. F., Greene, M. R., \& Oliva, A. (2011). Disentangling scene content from spatial boundary: Complementary roles for the parahippocampal place area and lateral occipital complex in representing real-world scenes. Journal of Neuroscience, 31, $1333-1340$.

Park, S., Intraub, H., Yi, D.-J., Widders, D., \& Chun, M. M. (2007). Beyond the edges of a view: boundary extension in human sceneselective visual cortex. Neuron, 54, 335-342.
Perez, D., Cook, S., \& Peterson, M. (2020). Prior experience alters the appearance of blurry object borders. Scientific Reports, 10, 5821.

Persaud, K., \& Hemmer, P. (2016). The dynamics of fidelity over the time course of long-term memory. Cognitive Psychology, 88, 1-21.

Serences, J. T., Ester, E. F., Vogel, E. K., \& Awh, E. (2009). Stimulusspecific delay activity in human primary visual cortex. Psychological Science, 20, 207-214.

Siegel, S., Byrne, A. (2017). Rich or thin? In B. Nanay (ed.), Current Controversies in Philosophy of Perception. Routledge.

Valenti, J. J., \& Firestone, C. (2019). Finding the "odd one out": Memory color effects and the logic of appearance. Cognition, 191, 103934.

$\mathrm{Xu}, \mathrm{Y}$. (2017). Reevaluating the sensory account of visual working memory storage. Trends in Cognitive Sciences, 21, 794-815.

Publisher's note Springer Nature remains neutral with regard to jurisdictional claims in published maps and institutional affiliations. 